Since the completion of the first draft sequence of the human genome, and with each subsequent new build, our understanding of the organization and regulation of the human genome continues to evolve. From the initial assemblies of sequences and the structural organization of individual chromosomes, we are now beginning to get glimpses of the complex networks that regulate the genome at multiple levels. Furthermore, recent investigations have identified new elements such as micro RNAs and genomic copy-number polymorphisms that are present throughout the genome and likely mediate various cellular and pathological processes. In addition, various genome projects have recently delivered high-quality sequences of multiple organisms and species ranging from viral and bacterial pathogens to Arabidopsis and canines. These represent a huge resource for studying genomes and for investigating and comparing biological systems at unprecedented levels of detail.

One of the key elements in this expansion of genomic and biological sciences has been the development of microarray technologies. The initial pioneering work with specific clone sets has been advanced with the availability of high-quality sequences for genomes of interest and the development of improved fabrication and analysis tools. Array content can now be readily customized to include entire genomes or regions of interest at variable coverage, providing high-density views of biological states and processes. Furthermore, since the publication of the first Chipping Forecast, the use of microarrays has expanded from gene-expression profiling to include a variety of applications such as array comparative genomic hybridization, alternate transcripts and microRNA profiling, and methylation and location analyses, as well as constitutive and somatic genotyping. In addition, the development of resources for studying functional genomics, such as siRNA and high-content cell-based assays, is expanding the application of microarrays. These technologies are providing tools for translating genomic sciences into biological and clinical investigations.
The ongoing advances in our understanding of genomic sciences and the development of new and more robust tools to investigate and analyze biological systems has led to an emphasis on analyzing biological systems at multiple levels. Thus, there is a need to integrate different types of data into a comprehensive 'systems' view. These include improved analytical and visualization tools and the ability to integrate different types of data into a comprehensive view of biological processes. These approaches are beginning to provide new and profound insights into human biology with the potential for new effective interventions in treating and preventing human diseases. The translation of microarray-based results to clinical applications challenges the technology at all levels. These include robust probe design, uniform sample preparation and increased reproducibility of array measurements. The recent advances in genomic sciences and array technologies are accelerating the translation of microarrays to clinical applications. For example, prognostic expression signatures for metastases have been identified in multiple studies across different array platforms and are now being validated in large prospective studies. These and other microarray-based studies offer enormous potential for improved health care in cancer and a variety of human diseases.

The Chipping Forecast has provided focused and timely reviews and summaries in the rapidly expanding world of microarray technology. The Perspectives in this current edition continue that tradition by providing insights from leading researchers who are pushing boundaries and challenging our understanding of biology at all levels. Agilent Technologies is committed to being an active participant, both as a supplier of array technology and a collaborator in innovative research, in the rapidly evolving world of genomic and biological sciences. We are proud to contribute to this third edition of The Chipping Forecast.

\author{
Michael T. Barrett \\ Agilent Laboratories \\ 3500 Deer Creek Road \\ Palo Alto, California 93404, USA
}

\title{
Agilent Technologies
}

